\title{
Quantum Structures of a Model-Universe: An Inconsistency with Everett Interpretation of Quantum Mechanics
}

\author{
J. Jeknić-Dugić*1, M. Dugić ${ }^{\dagger}$, A. Francom ${ }^{\$}$ \\ *Department of Physics, Faculty of Science, Niš, Serbia \\ ${ }^{\dagger}$ Department of Physics, Faculty of Science, Kragujevac, Serbia \\ \$Austin, TX 78748, USA
}

\begin{abstract}
We observe a Quantum Brownian Motion (QBM) Model Universe in conjunction with recently established Entanglement Relativity and Parallel Occurrence of Decoherence. The Parallel Occurrence of Decoherence establishes the simultaneous occurrence of decoherence for two mutually irreducible structures (decomposition into subsystems) of the total QBM model universe. First we find that Everett world branching for one structure excludes branching for the alternate structure and in order to reconcile this situation branching cannot be allowed for either of the structures considered. Second, we observe the non-existence of a third, "emergent structure", that could approximate both structures and also be allowed to branch. Ultimately we find unless world-branching requires additional criteria or conditions, or there is a privileged structure, that we provide a valid model that cannot be properly described by the Everett Interpretation of Quantum Mechanics.
\end{abstract}

Keywords Everett Interpretation, Entanglement Relativity, Quantum Brownian Motion, Quantum Decoherence

\footnotetext{
${ }^{1}$ Email: jjeknic@pmf.ni.ac.rs
} 


\section{Introduction}

"Today, it is often said that in Everettian quantum theory the notion of parallel 'worlds' or 'universes' applies only to the macroscopic worlds defined (approximately) by decoherence. Formerly, it was common to assert the existence of many worlds at the microscopic level as well. Without entering into any controversy that might still remain about this, here for completeness we shall address the Claim for both 'microscopic' and 'macroscopic' cases." ( Valentini 2010)

As the Everettian quantum theory claims to be a valid interpretation of the universally valid quantum mechanics, there should be no known model in which it fails to perform.

Using the Quantum Brownian Motion (QBM) model (Giulini et al 1996; Breuer and Petruccione 2002; Caldeira and Leggett 1985; Romero and Paz 1997) as a "model universe", Entanglement Relativity (ER) (Dugić 1999; Dugić and Jeknić 2006; Ciancio et al 2006; Dugić and Jeknić-Dugić 2008; de la Torre et al 2010; Harshman and Wickramasekara 2007; Jeknić-Dugić and Dugić 2008; Terra Cunha et al 2007; Tommasini et al 1998), and the Parallel Occurrence of Decoherence (POD) (Dugić and Jeknić-Dugić 2012), we will demonstrate for the first time using these methods, that Everett interpretation fails to properly describe a physically relevant decoherence model.

The QBM model affords us the opportunity to avoid getting involved with extending or arguing in the realm of interpretation because firstly it is a nontrivial model universe (a model of an isolated quantum mechanical system) referring to a realistic and mathematically well-defined physical situation, and secondly, the QBM model is paradigmatic for the Everett Interpretation ( S. Saunders 2010; Wallace 2010; Hartle 2010): as it directly implements the quasi-classical dynamics of the decoherence-selected basis of wavepackets approximately localized in both position and momentum of the open system (of the Brownian particle).

We consider a pair of structures (decompositions into subsystems), formally denoted $1+2$ and $S^{\prime}+E^{\prime}$, of the model universe. The structures are mutually related by the proper Linear canonical transformations (LCTs). This kind of restructuring the Universe is not new, see e.g. Saunders (2010), and Kent (2012). However, our focus goes beyond these general considerations in that it is devoted to the mutually irreducible structures, $1+2$ and $S^{\prime}+E^{\prime}$. This irreducibility of the structures provides the first main point of our consideration: For the model considered, the Everett branching for one structure excludes Everett branching for the other structure. In order to reconcile the two, we conclude that Everett branching cannot take place for 
any of the structures.

Modern Everett interpretation assumes there need not be branching for the "microscopic" model of a composite system. Rather, some effective, "emergent" structure (describable by the "higher level ontology") is expected to branch Wallace $(2010,2012)$. So, our first finding may not be relevant.

However, for the QBM model, the second main point of our considerations reads: we do not find physical degrees of freedom that might support the emergentism of modern Everett interpretation. Thereby we are forced to conclude that the Everett interpretation cannot properly describe the QBM universe-model.

A possible way out of this inconsistency with Everett theory might be to choose one structure as physically relevant (preferred), at the expense of rejecting the alternate structure as physically irrelevant, artificial.

In the absence of a physically sound and clear criterion for making the choice of the preferred universe structure, we finally conclude that there is a physically relevant model that cannot be properly described by the Everett interpretation of quantum mechanics.

In Section 2 we introduce the concept of entanglement relativity. In Section 3 we briefly present the recently obtained parallel occurrence of decoherence for quantum Brownian Motion (Dugić and Jeknić-Dugić 2012). Section 4 provides the main result of our paper. Section 5 is discussion and we conslude in Section 6.

\section{Entanglement relativity}

Below, we will discuss Entanglement Relativity (ER) as a subtle, and perhaps often overlooked aspect of the universally valid quantum mechanics. With an eye towards this subtlety, references (Dugić 1999; Dugić and Jeknić 2006; Ciancio et al 2006; Dugić and Jeknić-Dugić 2008; de la Torre et al 2010; Harshman and Wickramasekara 2007; Jeknić-Dugić and Dugić 2008; Terra Cunha et al 2007; Tommasini et al 1998) demonstrate how ER appears in quantum mechanics related articles. For the sake of clarity we will now highlight and analyze its important aspects.

The hydrogen atom is defined as a bipartite system "electron + proton $(e+p)$ ". However, in practice it is recognized as a pair "center of mass + relative coordinates $(C M+R)$ ". These two atomic decompositions (structures) are mutually linked by the well known linear canonical transformations (LCTs) which introduce $C M$ and $R$ to the atom. The relevant LCTs allow for the "separation of variables" and for the exact solution to the Schrödinger equation in the $C M+R$ decomposition. The related quantum state (while neglecting the atomic spin) is in tensor-product form $|\chi\rangle_{C M}\left|n l m_{l}\right\rangle_{R}$, where 
$n, l, m_{l}$ are the well known numbers from quantum theory for the hydrogen atom.

On the other hand, the Coulomb interaction between $e$ and $p$ leads to the conclusion that the pair $e+p$ must be in an entangled state of the form $\sum_{i} c_{i}|i\rangle_{e}|i\rangle_{p}$. Bearing in mind that $e+p=$ atom $=C M+R$, the universally valid quantum mechanics implies the following equality (for an instant of time):

$$
\sum_{i} c_{i}|i\rangle_{e}|i\rangle_{p}=|\chi\rangle_{C M}\left|n l m_{l}\right\rangle_{R}
$$

Dynamically: the $R$-system's state is stationary (multiplied by $\exp \left(-\imath t E_{n} / \hbar\right)$ ), while the $C M$-system's state may be e.g. a wave packet freely evolving in time.

Eq. (1) is paradigmatic for ER in that a change of the spatial degrees of freedom of a composite system typically results in a formal change of the composite system's quantum state. There is entanglement for every instantaneous quantum state of a composite quantum system and the very concept of entanglement is relative.

In a more abstract form eq. (1) reads as follows, where, of course, $1+2=$ $C=S+S^{\prime}:$

$$
\sum_{i} c_{i}|i\rangle_{1}|i\rangle_{2}=|\chi\rangle_{S}|\psi\rangle_{S^{\prime}}
$$

Then, one may undertake the task of kinematically transforming the left hand side into the right hand side of eq. (2), and vice versa. Generally, this is a formidable task not yet very well known. However, for some models (Breuer and Petruccione 2002; Caldeira and Leggett 1985; Romero and Paz 1997; Dugić and Jeknić 2006; de la Torre et al 2010), we already know about the validity of eq. (2) as a corollary of the universally valid quantum mechanics.

Equation (2) applies to a system $C$ of arbitrary complexity. To illustrate, one can first directly generalize ER as follows:

$$
\sum_{p} \alpha_{p}\left|\varphi_{p}\right\rangle_{1}\left|\Phi_{p}\right\rangle_{2}=\sum_{k} \beta_{k}\left|\mu_{k}\right\rangle_{S}\left|\nu_{k}\right\rangle_{S^{\prime}}
$$

Now, two remarks are in order regarding eq. (3). First, every subsystem of $C\left(1 ; 2 ; S ; S^{\prime}\right)$ may bear its own structure and related internal entanglement. Second, the above expressions equally refer to the cases when certain subsystems (e.g. the atomic spin, or the system's environment) are neglected or implicit or that are not yet known. Thereby ER eq. (2) equally addresses the hydrogen atom as well as the quantum Universe. For the hydrogen atom 
in a non-relativistic frame eq. (2) is precise, while for "the Universe" the expression of eq. (2), and likewise of eq. (3), assumes that further decomposing of universal subsystems is possible.

Eq. (2) reveals the presence of nontrivial non-negligible quantum entanglement in a composite quantum system relative to the pertinent degrees of freedom. We do not account for the change in entanglement due to a change of the reference frame (Gingrich and Adami 2002). ER otherwise is effectively ubiquitous.

Entanglement Relativity therefore is a descriptive name for that there is always an entangled form of a composite system's state. In its kinematical form eq. (2), ER directly points out inconsistency in the original Everett's "relative states" interpretation Everett (1957). A related dynamical consideration easily points out inconsistency in the modern Everett interpretation. The arguments will be given in Section 4 .

\section{Parallel occurrence of decoherence for irreducible structures of the model universe}

The occurrence of decoherence for the Universe is a plausible conjecture, an extrapolation of the existing (typically very simple) models of the decoherence theory. While there is progress in describing ever more-complex systems by decoherence, the truly complex systems, such as the Universe, are as yet out of reach. Therefore there is no alternative to modeling the Universe except but to employ relatively simple models.

Nevertheless, considering simple models does not decrease the importance of the conclusions obtained as the Everett Interpretation has pled to full universality, that is, to model-independence. In principle, this makes the simple models equally useful for drawing general conclusions within the Everett Interpretation, cf. e.g. Barvinsky and Kamenshchik (1995).

Bearing that in mind, we examine the well-known Quantum Brownian Motion (QBM) model (Giulini et al 1996; Breuer and Petruccione 2002; Caldeira and Leggett 1985; Romero and Paz 1997). First, it is a nontrivial decoherence model referring to a realistic physical situation. Second, the QBM model directly distinguishes the Gaussian states as the decoherence-selected "preferred states" of paramount importance for the Everett Interpretation:

"In contrast, states well localized in phase space-wavepackets-reliably decohere, and even though elements of a superposition, evolve autonomously from each other for a wide class of Hamiltonians. With respect to states like these, Ehrenfest's theorem takes on a greatly strengthened form. But decoherence in this sense is invariably approximate; it is never an all-or-nothing thing." (Saunders 2010) 


\subsection{Definition of the structures. Irreducibility}

Consider a pair of systems, 1 and 2. The 1 system is one dimensional, i.e. described by the position and the momentum observables, $x_{1}$ and $p_{1}$, respectively; the commutator, $\left[x_{1}, p_{1}\right]=\imath \hbar$. The 2 system is many-particle. The constituent particles described by the respective position and momentum observables, $x_{2 i}$ and $p_{2 i}$; with the commutator $\left[x_{2 i}, p_{2 j}\right]=\delta_{i j} \imath \hbar, i, j=1,2, \ldots N$. The composite system, $C$, is defined, $C=1+2$.

Now we introduce alternate structure of $C$. We do this by employing the proper LCTs that introduce a new pair of systems, $S^{\prime}$ and $E^{\prime}$. with the respective conjugate observables, $X_{S^{\prime}}$ and $P_{S^{\prime}}$, and $\rho_{E^{\prime} \alpha}$ and $p_{E^{\prime} \alpha}$. Again, we assume the new $S^{\prime}$ system is one-dimensional. Of course, $1+2=C=S^{\prime}+E^{\prime}$.

Every observable $x_{1}, x_{2 i}$ is assumed to be a linear combination of the alternate observables, $X_{S^{\prime}}$ and $\rho_{E^{\prime} \alpha}$, and vice versa; e.g., $X_{S^{\prime}}=\sum_{i=1}^{N+1} c_{i} x_{i}$, $\rho_{E^{\prime} \alpha}=\sum_{i} b_{\alpha i} x_{i}, \alpha=1,2, \ldots, N$. This makes the two structures, $1+2$ and $S^{\prime}+E^{\prime}$, mutually irreducible.

The irreducibility means (for some details see Section 4.2): a) that the $S^{\prime}$ (likewise the $E^{\prime}$ ) system cannot be decomposed or partitioned in to the 1 and 2 system and vice versa, and furthermore b) the degree of freedom of the 1 system (of the $S^{\prime}$ system), is a linear combination of all of the degrees of freedom of the $S^{\prime}$ and $E^{\prime}$ (of the 1 and 2) systems. Consequently no measurement of the 1 system represents a measurement of the $S^{\prime}$ system, and vice versa. Finally, c) the probability density of one subsystem does not yield a probability density for any other subsystem of the alternate structure.

The points (a)-(c) directly give rise to: (i) by very definition, every subsystem, $1,2, S^{\prime}, E^{\prime}$, belongs to only one structure, either to $1+2$ or to $S^{\prime}+E^{\prime}$; (ii) by very definition, both the 1 and $S^{\prime}$ systems are one-dimensional and cannot exchange particles with the rest, 2 and $E^{\prime}$; (iii) due to (b) and (c), a local observer [belonging either to $1+2$ or to $S^{\prime}+E^{\prime}$ ] cannot obtain any information about the $S^{\prime}$ system based on the information about the 1 system, and vice versa; (iv) due to (b) and (c), there is not any information flow between the subsystems belonging to the different structures.

Of course, we do not claim universal validity of the above (a)-(c), i.e. of (i)-(iv). There easily can be constructed mutually reducible structures for which some of these need not apply.

\subsection{Parallel decoherence for the QBM model}

We briefly present the results of Dugić and Jeknić-Dugić (2012). We borrow notation from Section 3.1. The QBM model considers a point-like particle 1 (or the particle's center-of-mass) interacting with harmonic-bath 
oscillators (system 2). The composite system $C=1+2$ is defined by the $C$ 's state-space $H_{1} \otimes H_{2}$ tensor-product structure and the total Hamiltonian:

$$
\begin{aligned}
& H=\frac{p_{1}^{2}}{2 m_{1}}+V\left(x_{1}\right)+\sum_{i}\left(\frac{p_{2 i}^{2}}{2 m_{2 i}}+\frac{m_{2 i} \omega_{i}^{2} x_{2 i}^{2}}{2}\right) \\
& \pm x_{1} \sum_{i} \kappa_{i} x_{2 i} \equiv H_{1}+H_{2}+H_{1+2}
\end{aligned}
$$

where the index $i$ enumerates the environmental particles, and the sign \pm is in accordance with the variations of the model contained in the literature. The physically relevant open system models are usually considered (cf. e.g. Breuer and Petruccione 2002): $V\left(x_{1}\right)=0$ for the free particle, or $V\left(x_{1}\right)=$ $m_{1} \omega^{2} x_{1}^{2} / 2$ for the harmonic oscillator.

The initial state $\rho_{C}$ of the pair $1+2$ is separable, $\rho_{C}=\rho_{1} \otimes \rho_{2 t h}$, while $\rho_{2 t h}$ means that the harmonic-bath environment is in thermal equilibrium. The general QBM theory (Giulini et al 1996; Breuer and Petruccione 2002; Caldeira and Leggett 1985; Romero and Paz 1997) states: The open system 1 is subject to decoherence induced by its environment 2, while related "pointer basis" (the robust, quasi-classical) states are the Gaussian states. The Gaussian state dynamics is very much like that which would be expected in a classical system: due to the large environment 2, decoherence effectively irreversibly destroys the linear superpositions of the Gaussian states of the system 1 ; the environment 2 effectively performs the approximate positionmeasurement for system 1. System 1, the "Brownian particle", exhibits quasi-classical behavior very much like the "classical Brownian particle". Therefore the composite $C$ system is a proper model-universe.

Now, we introduce another structure, $S^{\prime}+E^{\prime}$, for the isolated composite system $C$ (Dugić and Jeknić-Dugić 2012). The Hilbert state space of $C$ is now factorized, $H=H_{S^{\prime}} \otimes H_{E^{\prime}}$. We first introduce the standard $C M$ (center of mass) and $R$ (relative positions) for the total system: $X_{C M}=\sum_{i} m_{i} x_{i} / \sum_{j} m_{j}$ and $r_{R \alpha}=x_{i}-x_{j}, \alpha \equiv(i, j)$, and $P_{C M}$ and $p_{R \alpha}$ represent the respective conjugate momentums. The Hilbert state space factorizes $H=H_{C M} \otimes H_{R}$.

For the $H_{1}+H_{2}$ part of the Hamiltonian eq. (4), i.e for the set of noninteracting particles, it is known (Mc Weeny 1978) that $H_{1}+H_{2}$ transforms as follows. The kinetic terms for all the new particles takes the standard form $p^{2} / 2 m$; the $C M$ system's mass is the total $S+E$ system's mass, $M$, while the $R$ system's constituent oscillators' masses are the reduced masses, $\mu_{\alpha}$ for the $\alpha$ th oscillator. There appears the so-called "mass polarization" term that bilinearly couples the momentums of the environmental oscillators. Physically, the new structure $C M+R$ consists of the $C M$ system, which does not interact with the $R$ system, and the $R$ system is a set of linearly coupled oscillators. 
When we take the interaction $H_{1+2}$ into account, one easily obtains: there appear additional quadratic terms (harmonic potentials) for the $C M$ system as well as for every $R$-system's oscillator, while there is a linear coupling between the position observables for the $C M$ and $R$-system's oscillators, of the same form as given in eq. (4). So, the $C M$ system is a harmonic oscillator even if it was not the case for the original open system 1 . There also appear bi-linear coupling of the position observables for the environmental oscillators. In effect, there is bilinear coupling of the position, as well as of the momentum observables for the environmental (for the $R$ 's) oscillators.

For the oscillators bi-linearly coupled via their position and/or momentums, one can always perform another linear variables-transformation in order to decouple the oscillators (Mc Weeny 1978). So, for the $R$ system we can introduce the new position observables (the normal modes), $\rho_{R \alpha}$, and the related conjugate momentums, $\pi_{R \alpha}$, for which the $R$-system becomes a set of mutually uncoupled oscillators. With the notation, $S^{\prime} \equiv C M$ and $E^{\prime} \equiv R$, the Hamiltonian eq. (4) acquires the form:

$$
\begin{aligned}
& H=\frac{P_{S^{\prime}}^{2}}{2 M}+\frac{1}{2} M \Omega^{2} X_{S^{\prime}}^{2}+\sum_{\alpha}\left(\frac{\pi_{E^{\prime} \alpha}^{2}}{2 \mu_{\alpha}}+\frac{1}{2} \mu_{\alpha} \nu_{\alpha}^{2} \rho_{E^{\prime} \alpha}^{2}\right) \\
& \pm X_{S^{\prime}} \sum_{\alpha} \sigma_{\alpha} \rho_{E^{\prime} \alpha} \equiv H_{S^{\prime}}+H_{E^{\prime}}+H_{S^{\prime}+E^{\prime}} .
\end{aligned}
$$

which is formally isomorphic with the form of eq. (4) for the original $1+2$ structure.

There are a number of details differentiating between the two structures, $1+2$ and $S^{\prime}+E^{\prime}$ (Dugić and Jeknić-Dugić 2012, Jeknić-Dugić et al 2013). Nevertheless, as it is well-known (Giulini et al 1996; Breuer and Petruccione 2002; Caldeira and Leggett 1985; Romero and Paz 1997), the occurrence of the Brownian effect is largely independent from the details distinguishing both models, eq. (4) and eq. (5). Particularly, the occurrence of decoherence (of the effective approximate position-measurement of the Brownian particle) is independent of the presence/absence of correlations (quantum or classical) in the initial state, of the strength of the interaction in the composite system "Brownian particle + harmonic-oscillators-environment", or on the so-called form of the "spectral density". The formal similarity between the two models, eq. (4) and eq. (5), allows the following conclusion on the parallel occurrence of decoherence (POD) (Dugić and Jeknić-Dugić 2012):

The unitary evolution of the initial state $\rho_{C}$ generated by the Hamiltonian $H$ gives, for the different structures of $C$ : For as much as System 1 represents the "Brownian Particle", in the $1+2$ decomposition, System $S^{\prime}$ represents the "Brownian Particle" for the $S^{\prime}+E^{\prime}$ decomposition. 
Physically, both structures bear the decoherence-induced quasiclassicality that is required in the foundations of modern Everett interpretation. So we have a model Universe $C$ with the two structures that are simultaneously and quasiclassically evolving in time, $1+2$ and $S^{\prime}+E^{\prime}$.

\section{The inconsistency}

It is worth repeating: Both structures, eq. (4) and eq. (5), are formally equal and mutually irreducible. Both structures bear a physically clear "system-environment split" with large environments capable of providing fast decoherence as an effectively irreversible process. As both Brownian particles, 1 and $S^{\prime}$, are elementary (one-dimensional), the number of degrees of freedom in 2 is equal to the number of the degrees of freedom in the $E^{\prime}$ environment. The initial state for both decompositions is the same, while being subject to ER, Section 2. The two decoherence processes (for 1 and for $S^{\prime}$ ) unfold simultaneously in time. The basis (the "preferred states") picked out by decoherence for both open systems is approximately e.g a "coherentstate" (a wavepacket) basis whose dynamics are quasi-classical in the sense that the behavior of those wavepackets approximates the behavior predicted for the classical Brownian particle.

\subsection{Non-branching of the structures}

Let us consider the dynamics of the $1+2$ structure. The decoherencepreferred states are known to be Gaussians (Giulini et al 1996; Breuer and Petruccione 2002; Caldeira and Leggett 1985; Romero and Paz 1997). Then one can write (cf. eq. (5) in Wallace 2010) for the universal state:

$$
|\Psi(t)\rangle_{C}=\int d x_{1} d p_{1} \alpha\left(x_{1}, p_{1}, t\right)\left|x_{1}, p_{1}\right\rangle_{1}\left|\epsilon\left(x_{1}, p_{1}\right)\right\rangle_{2},
$$

where $\left|x_{1}, p_{1}\right\rangle_{1}$ is a "coherent state" for the 1 system in correlation with the approximately orthogonal environmental states, $\left|\epsilon\left(x_{1}, p_{1}\right)\right\rangle_{2}$. In the presence of decoherence, Section 3.2, $\left|\alpha\left(x_{1}, p_{1}, t\right)\right|^{2}$ evolves, to a good approximation, like a classical probability density on phase space for the 1 open system. Owing to the correlations in Eq. (6), one can define a set of consistent histories for the total system $1+2$. One such history for the time instants $t_{\circ}<t_{1}<t_{2} \ldots$ :

$$
\begin{aligned}
& \left.\left.\left|x_{1}\left(t_{\circ}\right), p_{1}\left(t_{\circ}\right)\right\rangle_{1}\left|\epsilon\left(x_{1}\left(t_{\circ}\right), p_{1}\left(t_{\circ}\right)\right)\right\rangle_{2} \rightarrow\left|x_{1}\left(t_{1}\right), p_{1}\left(t_{1}\right)\right\rangle_{1} \mid \epsilon\left(x_{1}\left(t_{1}\right)\right), p_{1}\left(t_{1}\right)\right)\right\rangle_{2} \\
& \left.\left.\rightarrow \mid x_{1}\left(t_{2}\right), p_{1}\left(t_{2}\right)\right)\right\rangle_{1}\left|\epsilon\left(x_{1}\left(t_{2}\right), p_{1}\left(t_{2}\right)\right)\right\rangle_{2} \ldots
\end{aligned}
$$


[defined with some probability], approximately represents dynamics of an Everet world.

The point to be strongly emphasized: Entanglement Relativity, Section 2, refers to every instant in time. A tensor-product state $\left|x_{1}\left(t_{i}\right), p_{1}\left(t_{i}\right)\right\rangle_{1}\left|\epsilon\left(x_{1}\left(t_{i}\right), p_{1}\left(t_{i}\right)\right)\right\rangle_{2}$ obtains entangled form for the alternate structure $S^{\prime}+E^{\prime}$ for practically every time instant $t_{i}, i=0,1,2, \ldots$. As a consequence, the set of mutually consistent histories for the $1+2$ structure is not "consistent" for the structure $S^{\prime}+E^{\prime}$. So Everett branching for the $1+2$ structure excludes the Everett branching for the $S^{\prime}+E^{\prime}$ structure.

Section 3.2 now suggests the roles of the two structures in this analysis can be inverted. More precisely, as the two structures are equally valid decompositions of the Universe, exchanging the roles of the structures in the above analysis leads us to the following observation: Everett branching for the $S^{\prime}+E^{\prime}$ structure excludes Everett branching for the $1+2$ structure.

In effect, an Everett branch (an Everett world) for one structure cannot last longer than the decoherence-induced branching for the alternate structure. Bearing in mind the fact that decoherence is a fast process, also for the model considered in Section 3.2 (Dugić and Jeknić-Dugić 2012), we find: Mutually exclusive yet simultaneous splitting processes for the two structures effectively result in the impossibility of World-Branching for both structures.

It is important to emphasize: Everett branching is not in conflict with either ER or POD, separately, it is not consistent with ER and POD together. On the other hand, both ER and POD are corollaries of the universally valid quantum mechanics. Therefore we conclude:

The main interpretational rule of branching of the Everett worlds is in conflict with the universally valid quantum mechanics for the QBM model of Section 3.

\subsection{In search of emergent structure for the QBM model}

Decoherence is typically studied starting from a fairly unprincipled choice of system-environment split. In this sense, decoherence is by its nature an approximate process and so the process of branching is likewise approximate. In other words (Wallace 2010) [our emphasis]: "...decoherence is an emergent process occurring within an already stated microphysics: unitary quantum mechanics. It is not a mechanism to define a part of that microphysics". This is the basis for the modern Everett interpretation that can be expressed as follows [our emphasis]:

"There is just a dynamical process-decoherence-whereby certain components of that state become dynamically autonomous of one another. Put another way: if each decoherent history is an emergent structure within the underly- 
ing microphysics, and if the underlying microphysics doesn't do anything to prioritize one history over another (which it doesn't) then all the histories exist. That is: a unitary quantum theory with emergent, decoherence-defined quasiclassical histories is a many-worlds theory." (Wallace 2010)

Within this new wisdom, one may suppose that there should be an emergent structure for the model-Universe $C$ that should branch. In other words: the structures $1+2$ and $S^{\prime}+E^{\prime}$ may be considered "microscopic" i.e. of no interest individually for branching. This can make the finding of Section 4.1 pointless.

To test whether the finding of Section 4.1 is pointless in this light, one should construct an emergent model-structure eligible for the branching.

In the absence of a general physical definition of "emergent properties" (i.e. of the "higher level ontology") of complex systems, cf. e.g. Auyang (1998), we are forced to speculate about the possible ways to obtain a branching-eligible structure for the QBM model of Section 3. To this end, we are able to detect only two possibilities. We find both of them inappropriate for defining an emergent QBM structure or for recognizing their decoherence induced dynamics to approximate each other.

We distinguish the following bases for emergentism. First, it is the dynamical exchange of particles between the "system" and the "environment" that encompasses the standard choice of the "dividing line" in the von Neumann sense (the von Neumann "chain"), von Neumann (1955). Second, one may suppose there is an alternate, third structure providing an emergent Brownian particle, $B$, for the pair of Brownian particles, 1 and $S^{\prime}$, Section 3.

To see that the first doesn't work for the QBM model is straightforward. Actually, both Brownian particles are one-dimensional and there is not, by definition, any possibility of exchanging particles of e.g. the 1 system with the environment 2 (or of the $S^{\prime}$ system with the environment $E^{\prime}$ ); of course, due to irreducibility of the two structures, exchange of the particles between the 1 system with the environment $E^{\prime}$ (i.e. of the $S^{\prime}$ system with 2 ) is not even defined. The variant that an environmental oscillator takes the role of Brownian particle is in principle also not allowed. In both models, Eq. (4) and Eq. (5), the environmental particles do not mutually interact and therefore there is not a properly defined environment for the variant-not to mention that this excludes the possibility (Giulini et al 1996; Breuer and Petruccione 2002; Caldeira and Leggett 1985; Romero and Paz 1997) that the 1 system is a "free particle" (not an oscillator). So the system-environment split cannot be altered for the QBM model.

The second option is a bit more subtle yet. To this end we justify the claims of Section 3.1: (1) obtaining information about one Brownian particle (e.g. 1) provides no information about the alternate one (e.g. the particle 
$\left.S^{\prime}\right)$; (2) there does not exist any observable, $X_{B}$, of the subsystem $B$ of the composite system $C$ that would approximate measurements of any pair of observables of the two Brownian particles, 1 and $S^{\prime}$. In effect, there is not any structure $B+E_{B}$ of $C$ that could be emergent for the structures $1+2$ and $S^{\prime}+E^{\prime}$.

Regarding the point (1), we first remind (cf. Section 3.1): the variables of the 1 (of the $S^{\prime}$ ) are linear functions of all the variables of the $S^{\prime}$ and $E^{\prime}$ (of the 1 and 2) systems. So local measurements performed on the $S^{\prime}$ system reveals nothing about the 1 system, and vice versa. More formally: $\operatorname{tr}_{2}|\Psi\rangle\langle\Psi|$ provides a probability density, $\rho\left(x_{1}, x_{1}^{\prime}\right)$, for the 1 system, and analogously for the $S^{\prime}$ system. It is obvious that $\rho\left(x_{1}, x_{1}^{\prime}\right)$ cannot in principle be used to define any probability density for $S^{\prime}$, and vice versa. e.g. The integral $\int \rho\left(x_{1}, x_{1}^{\prime}\right) \Pi^{\otimes \alpha} d \rho_{E^{\prime} \alpha}$ is not a probability density for the $S^{\prime}$ system. Furthermore, linear dependence of the observables $x_{1}$ and $X_{S^{\prime}}$ makes the "tracing out" e.g. of the form $\int \rho\left(x_{1}, X_{S^{\prime}}\right) d X_{S^{\prime}}$ ill-defined. Thereby one can say the Brownian particles, 1 and $S^{\prime}$, belonging to the mutually irreducible structures, are mutually information-theoretically separated. The two decoherence processes, for the 1 system and for the $S^{\prime}$ system, cannot approximate each other, nor is there any information flow between 1 and $S^{\prime}$. The conclusion refers to every subsystem, including the observer of a structure. An observer belonging to the $1+2$ structure is a subsystem of the 2 environment, while the observer belonging to the $S^{\prime}+E^{\prime}$ structure is a subsystem of the $E^{\prime}$ environment. Then e.g. by exchanging the $x_{1}$ above by the observer's position, $x_{o b s}$, we conclude: the alternative structure's (the $S^{\prime}+E^{\prime}$ structure's) subsystems are information-theoretically separated from the observer belonging to the alternate (i.e. to the $1+2$ ) structure-and vice versa. In a picturesque way, we can say, that only the set of the degrees of freedom of the structure the observer belongs to is consistent with the operation of the brain of the observer.

The arguments for point (1) apply to point (2). As the only probability density that can provide probability density for the arbitrary subsystem of $C$ is the universal state, $|\Psi\rangle$, there is not any subsystem's (B's) probability density, $\rho\left(X_{B}, X_{B}^{\prime}\right)$, that could provide probability density for both the 1 and the $S^{\prime}$ system. e.g. The definition $X_{B}=f\left(x_{1}, X_{S^{\prime}}\right)$ gives rise to the probability density $\rho\left(X_{B}, X_{B}^{\prime}\right)=\rho\left(x_{1}, x_{1}^{\prime}, X_{S^{\prime}}, X_{S^{\prime}}^{\prime}\right)$, which, as emphasized above, cannot provide the probability densities $\rho\left(x_{1}, x_{1}^{\prime}\right)$ or $\rho\left(X_{S^{\prime}}, X_{S^{\prime}}^{\prime}\right)$ by integrating over $X_{S^{\prime}}$ and $x_{1}$, respectively. So, there is not any observable of the $B$ system whose measurement might approximate simultaneous measurement of any pair of observables for the two Brownian particles, 1 and $S^{\prime}$. Physically, this means that we cannot imagine a third system $B$, that undergoes Brownian-motion-like dynamics and can still approximately describe 
both "microscopic" particles, 1 and $S^{\prime}$.

As we cannot recognize any other basis for emergentism, we are forced to conclude that the above-distinguished inconsistency between the QBM model and the modern Everett interpretation remains intact.

\subsection{Sufficiency of decoherence for branching}

In the absence of an emergent model-structure encompassing the structures of Section 3, we consider and answer the following: Is the QBM model a proper subject of the Everett Interpretation?

To this end, we first emphasize: the standard QBM is a (paradigmatic theoretical) decoherence model pertaining to the realistic macroscopic situation of "Brownian motion". Second, there are not any structural phenomenological facts about Brownian motion that go beyond the standard QBM model (Giulini et al 1996; Breuer and Petruccione 2002; Caldeira and Leggett 1985; Romero and Paz 1997)-no need for any "emergent" Brownian particle.

Bearing this in mind, the possibility that the structures considered are not susceptible to the Everett interpretation directly raises the following foundational question: Is decoherence sufficient for Everett Branching ? If it is, then the conclusion of Section 4.2 is unavoidable. If not, then Section 4.2 suggests an additional requirement for branching, i.e. for completeness of the Everett interpretation is needed. e.g. One may require some amount of "complexity" of the composite system to be the subject of the modern Everett interpretation. Certainly, then the range of applicability of the modern Everett interpretation shrinks, as distinct from the competitive interpretations. As the "additional requirement" is not a part of the present state of the art in the field, and is not of interest for the QBM model, we will not elaborate on this any further. So we finally return to the conclusion of Section 4.2 .

\section{Discussion}

Our arguments are technical. We use Entanglement Relativity and the Parallel Occurrence of Decoherence as corollaries of the universally valid quantum mechanics. Thereby, every sound interpretation must not be in conflict with them. We do not enter any open issue of the Everett interpretation, such as the choice of the preferred pointer basis or the origin of probability. To this end, our considerations are non-interpretational. Essential for our argument is the use of ER and POD together, not separately. Nevertheless, ER+POD is not sufficient for our argument. Actually, we apply ER+POD on a pair of very special, mutually irreducible structures of the model universe. Only having the irreducible structures, $1+2$ and $S^{\prime}+E^{\prime}$, 
can we argue non-existence of an emergent Brownian particle $B$, for the pair of Brownian particles, 1 and $S^{\prime}$, and thereby point out inconsistency with the Everett interpretation.

In physics, "emergence" is usually linked with the "collective" degrees of freedom of a composite system. E.g. the phonons refer to a chain of oscillators as a whole. Knowledge about the phonons-system state provides information (via some calculation) about every original oscillator, and vice versa. However, in our considerations we ask about "emergence" for the pair 1 and $S^{\prime}$, which do not bear any structure of their own. We show that, as long as we require some information about 1 and $S^{\prime}$ to be derivable from the information about $B$, then there's no room for emergent Brownian particle $B$. If we do not require derivability of information about 1 and $S^{\prime}$, it is not clear in which physical sense we can claim $B$ is emergent for 1 and $S^{\prime}$. Therefore, the two Brownian particles 1 and $S^{\prime}$ should be considered as literally presented in Section 3-as mutually irreducible and simultaneously evolving in time according to the general rules of the quantum Brownian motion model (Giulini et al 1996; Breuer and Petruccione 2002; Caldeira and Leggett 1985; Romero and Paz 1997; Dugić and Jeknić-Dugić 2012).

It appears that the only remaining way to avoid the inconsistency with the Everett theory may be to claim physical relevance (e.g. physical reality, in whatever sense) of only one structure, e.g. of the $1+2$ structure, at the expense of rejecting the other (i.e. the $S^{\prime}+E^{\prime}$ ) structure (and the related branching process) non-physical, artificial. However, this does not seem a very promising strategy. The only assumption of our considerations (besides the universal validity of quantum mechanics) is that the Universe is an $e x$ actly isolated ("closed") system, i.e. that there is not any observer outside the Universe. Bearing this in mind, claiming the preferred structure of the Universe does not bear firm physical support yet. In the words of Zanardi (2001):

"Without further physical assumption, no partition has an ontologically superior status with respect to any other."

as well as of Halliwell (2010):

"However, for many macroscopic systems, and in particular for the universe as a whole, there may be no natural split into distinguished subsystems and the rest, and another way of identifying the naturally decoherent variables is required."

In the absence of a clear physical rule for making a choice of the preferred structure of the model-Universe, Section 3, we seem obliged to return to our conclusion on insufficiency of the Everett interpretation to properly describe the standard quantum Brownian motion model. 


\section{Conclusion}

We apply Entanglement Relativity and the parallel occurrence of decoherence to the standard quantum Brownian motion (QBM) setup. For the QBM model we distinguish a pair of special, mutually irreducible structures (decompositions into subsystems) for which neither Everett worlds branching is allowed nor can we detect any "emergent" Brownian particle that could branch. So unless world-branching requires additional criterion or condition, or there is a privileged structure of the model universe, we conclude that the universally valid and complete quantum mechanics does not support the Everett interpretation for the QBM-model structures considered.

Acknowledgements JJD and MD acknowledge financial support from the Ministry of Science Serbia under contract no 171028, and partially to the COST Action MP1006.

\section{REFERENCES}

Auyang, S. 1998 Foundations of Complex-system Theories. Cambridge: Cambridge University Press.

Barvinsky, A. O. \& Kamenshchik, A. Yu. 1995 Preferred basis in quantum theory and the problem of classicalization of the quantum Universe. Phys. Rev. D 52, 743-757.

Breuer, H.-P. \& Petruccione, F. 2002 The Theory of Open Quantum Systems. Oxford: Clarendon Press.

Caldeira, A. O. \& Leggett, A. J. 1985 Influence of damping on quantum interference: An exactly soluble model. Phys. Rev. A 31, 1059-1066.

Ciancio E., Giorda P. \& Zanardi P. 2006 Mode transformations and entanglement relativity in bipartite Gaussian states. Phys. Lett. A 354, 274-280.

De la Torre, A. C. et al 2010 Entanglement for all quantum states. Europ. J. Phys. 31, 325-332.

Dugić M. 1999 What is "system": the arguments from the decoherence theory. arXiv:quant-ph/9903037v1.

Dugić, M. \& Jeknić, J. 2006 What is "System": Some DecoherenceTheory Arguments. Int. J. Theor. Phys. 45, 2249-2259.

Dugić, M. \& Jeknić-Dugić, J. 2008 What Is "System": The InformationTheoretic Arguments. Int. J. Theor. Phys. 47, 805-813.

Dugić, M. \& Jeknić-Dugić, J. 2012 Parallel Occurrence of Decoherence in Composite Quantum Systems. Pramana: J. Phys. 79, 199-211.

Everett, H. 1957 "Relative state" formulation of quantum mechanics. Rev. Mod. Phys. 29, 454-462. 
Gingrich, R. M. \& Adami, C 2002 Quantum Entanglement of Moving Bodies. Phys. Rev. Lett. 89, 270402.

Giulini, D., Joos, E., Kiefer, C., Kupsch, J., Stamatescu, I.-O.\& Zeh, H. D. 1996 Decoherence and the Appearance of a Classical World in Quantum Theory. Berlin: Springer.

Halliwell, J. 2010 Emergence of Hydrodynamic Behaviour. In Many Worlds? Everett, Quantum Theory, and Reality (ed., S. Saunders, J. Barrett, A. Kent, \& D. Wallace), pp. 99-117. Oxford: Oxford University Press.

Harshman, N. L. \& Wickramasekara, S. 2007 Galilean and Dynamical Invariance of Entanglement in Particle Scattering. Phys. Rev. Lett. 98, 080406.

Jeknić-Dugić, J. \& Dugić, M. 2008 Multiple System-Decomposition Method for Avoiding Quantum Decoherence. Chin. Phys. Lett. 25, 371-374.

Jeknić-Dugić J. Arsenijević M, Dugić M., 2013 Quantum Structures: A View of a Quantum World. Saarbrucken: Lambert Academic Publishing; also available as arXiv:1306.5471 [quant-ph].

Kent, A. 2012 Real World Interpretations of Quantum Theory. Found. Phys. 42, 421-435.

Mc Weeney R. 1978 Methods of Molecular Quantum Mechanics. New York: Academic Press

Romero, L. D. \& Paz, J. P. 1997 Decoherence and initial correlations in quantum Brownian motion. Phys. Rev. A 55, 4070-4083.

Saunders, S. 2010 Many Worlds? An Introduction. In Many Worlds? Everett, Quantum Theory, and Reality (ed., S. Saunders, J. Barrett, A. Kent, \& D. Wallace), pp. 1-52. Oxford: Oxford University Press.

Terra Cunha, M. O., Dunningham, J. A. \& Vedral, V. 2007 Entanglement in single-particle systems. Proc. R. Soc. A 463, 2277-2286.

Tommasini, P., Timmermans, E. \& Piza, A.F.R.D. 1998 The hydrogen atom as an entangled electron-proton system. Am. J. Phys. 66, 881-885.

Valentini, A. 2010 De Broglie-Bohm Pilot-Wave Theory: Many Worlds in Denial?. In Many Worlds? Everett, Quantum Theory, and Reality (ed., S. Saunders, J. Barrett, A. Kent, \& D. Wallace), pp. 476-509. Oxford: Oxford University Press.

von Neumann, J. 1955 Mathematical foundations of quantum mechanics. Princeton: Princeton University Press.

Wallace, D. 2010 Decoherence and Ontology. In Many Worlds? Everett, Quantum Theory, and Reality (ed., S. Saunders, J. Barrett, A. Kent \& D. Wallace), pp. 53-72. Oxford: Oxford University Press.

Wallace, D. 2012 The Emergent Multiverse: Quantum Theory according to the Everett Interpretation. Oxford: Oxford University Press. 
Zanardi, P. 2001 Virtual Quantum Subsystems. Phys. Rev. Lett. 87, 077901. 